doi:10.1093/aob/mcs282

Published electronically: 28 December 2012

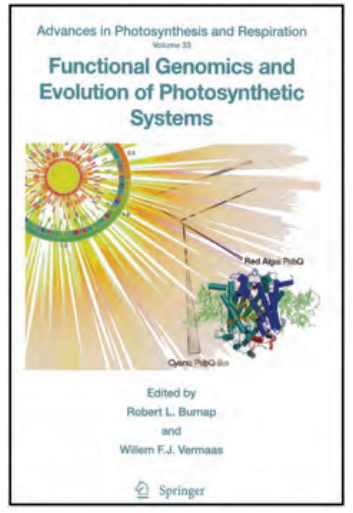

\section{Functional genomics and evolution of photosynthetic systems}

Robert L. Burnap, Willem F. J.

Vermaas. eds. 2012.

Springer. €223.63 (hardback). pp. 392.

This book, which is volume 33 in Springer's series 'Advances in photosynthesis and respiration', represents an excellent update of current thinking about the evolution of photosynthetic systems. It covers almost all aspects of photosynthesis: starting with the role of photosynthetic bacteria in diverse environments, giving examples of the huge progress in our understanding of evolution from the increasing amount of genome information, describing new aspects of integration of the photosynthetic organelle into eukaryotic organisms, and, finally, showing the progress in photosynthetic research from molecular and structural information. The book's editors, two leading scientists in the field of photosynthesis working mostly on cyanobacteria as model organisms, have been able to bring together a number of outstanding experts. I was very happy to see that this book is dedicated to Dr Teruo Ogawa, retired Professor from Nagoya University, Japan, who made fundamental contributions for example to the understanding of inorganic carbon utilization and the various roles of the NDH complexes, which integrate photosynthesis and respiration in cyanobacteria.

It is generally accepted that oxygenic photosynthesis evolved in ancient cyanobacteria and was conveyed to eukaryotic phototrophs by an endosymbiotic engulfment of a cyanobacterium-like prokaryote, leading to the evolution of plastids. The increasing number of genome sequences from diverse photosynthetic organisms and also structural information about photosynthetic systems, as reviewed in detail in this interesting book, support this view and add many new facets as to how and why certain developments occurred. However, despite this great progress, many questions remain open. For example, it is still debated how oxygenic photosynthesis using two photosystems evolved. Two possible scenarios are discussed: it might have occurred after combining photosystem II from purple bacteria and photosystem I from green sulfur bacteria or, alternatively, the two photosystems could have developed after gene duplication in anoxygenic protocyanobacteria. In this regard, it is of great interest to find new types of prokaryotic phototrophs, which might have retained ancient features. In the first four chapters, new insights into photosynthesis are reviewed coming from those studies with cyanobacteria and anoxic green bacteria in their natural environment. Based on the vastly increased amount of genome information, it has become possible to search for the existence, and analyse the performance, of phototrophic bacteria in extreme environments. For example, metagenomics and metatranscriptomics have revealed niche adaptation of thermophilic cyanobacteria. These strains from Yellowstone National Park build an extra clade of cyanobacteria close to the base of the cyanobacterial radiation. Such attempts have also led to the identification of a new group of anoxygenic green bacteria, the chlorophototrophic Acidobacteria. The genomic information collected for these bacteria shed new light on evolution of chlorophyll biosynthesis and ancient carbon fixation pathways. Finally, the phylogenetic relationship between anoxygenic green sulfur bacteria and cyanobacteria is discussed using proteins involved in pigment synthesis and antenna proteins. These analyses suggest that photosystem-Icontaining anoxygenic bacteria are older than cyanobacteria. Marine Synechococcus species are picoplanktonic cyanobacteria that live in the central oceans and are of global importance. Genome information and expression studies revealed how these cyanobacteria could adapt to essentially nutrientfree regions.

The next chapters deal with methods for the functional analysis of photosynthesis in different model organisms. New genetic tools to analyse photosynthesis-related genes are discussed. With the cyanobacterial model strain Synechococcus elongatus PCC 7942, the establishment and screening of a whole-genome mutant library is presented, which has allowed the identification of new components of the circadian clock. Among eukaryotes, the green alga Chlamydomonas reinhardtii is one of the most widely used models for photosynthesis research. The authors of Chapter 6 explain the beauty of this model and its flexibility in the electron transport chain under fluctuating environments using transcriptomics. Genome mining with Chlamydomonas, cyanobacteria and plants has allowed the definition of genes coding for yet unknown proteins that are most likely associated with the functioning of photosynthesis. Among plants, Arabidopsis thaliana became the most prominent model in the genome area. The whole array of 'omics' technologies for the analysis of photosynthetic functions in land plants including future directions is discussed. An important point of evolution of photosynthesis is its integration into the compartmented eukaryotic cells. Interaction of the chloroplast and other organelles needs a high number of transporters and signalling, which is one of the least understood aspects.

The last seven chapters each deal with special photosynthetic systems using genomic, molecular and structural data to shed more light on their evolution and function. In this context, the variety of known carbon fixation pathways is reviewed, including new potential modes of carbon dioxide fixation identified from genome information. Cyanobacteria restrict Rubisco to a special prokaryotic compartment, the carboxysome, which is part of an efficient carbon-concentrating mechanism. The evolution and dynamics of carboxysomal proteins are discussed in a later chapter using newly identified components and structures. Two chapters deal with the structure and evolution of antenna proteins in purple bacteria and cyanobacteria and discuss their roles in light harvesting, the regulation of photosynthesis and light protection. Based on recently available structural information, the evolution of photosystem I, including its adaptation to oxygen in the evolving cyanobacterial cell, is presented in much detail. The authors define critical steps to change the structure of photosystem I centres from the precursor, i.e. structures of photosystem I from green bacteria are compared to the contemporary photosystem I of cyanobacteria in which the iron-sulphur 
centres have to be protected against oxidation. Moreover, photosystem II and its extrinsic parts are shown in impressive structural models. The consequences of sequence changes are discussed in terms of function and adaptation to different light regimes. Special attention is directed to the lumenal proteins protecting the water-splitting complex in cyanobacteria, algae and plants. The final chapter deals with hydrogenases. These enzymes are associated with photosynthesis in prokaryotic phototrophs. Among cyanobacteria, one hydrogenase cooperates with nitrogenase during nitrogen fixation and another, the bidirectional hydrogenase, is discussed as a valve to release electrons from the over-reduced photosynthetic electron transport chain. Recent structural and genome information on these enzymes are summarized, highlighting potential avenues for the exploitation of this new knowledge in the production of biohydrogen as a 'green' energy source.

In conclusion, this book is an excellent synopsis of current thinking about evolution of photosynthesis on different system levels. It provides a superb collection of examples showing how the recent development of technologies has allowed long-debated questions about the adaptation of photosynthetic complexes for new environments to be answered. It also gives many examples how this progress in our knowledge can be used more and more to exploit natural biological systems for green energy production. It gives an excellent introduction into the recent literature and the controversial debate on the evolution of the variety of photosynthetic systems we know today. The book is not only highly relevant for research scientists working on the different aspects of photosynthesis, but also for graduate students interested in molecular plant biology, and it can be strongly recommended.

Martin Hagemann 